\title{
The usefulness of ICG video angiography in the surgical treatment of superior cluneal nerve entrapment neuropathy
}

\author{
Technical note
}

\author{
Kyongsong Kim, M.D., Рh.D., ${ }^{1}$ Toyohiko Isu, M.D., Рh.D., ${ }^{2}$ YAsuhiro Chiba, M.D., Рh.D., ${ }^{2}$ \\ Daijiro Morimoto, Ph.D.,M.D. , ${ }^{3}$ Seiji Ohtsubo, D.D.S., Ph.D., ${ }^{4}$ \\ Mitsuo Kusano, M.D., Ph.D. ${ }^{5}$ Shiro Kobayashi, M.D., Ph.D., ${ }^{1}$ \\ and Akio Morita, M.D., Ph.D. ${ }^{6}$ \\ ${ }^{I}$ Department of Neurosurgery, Chiba Hokuso Hospital, Nippon Medical School. Chiba, Japan; Departments \\ of ${ }^{2}$ Neurosurgery, ${ }^{4}$ Dentistry, Oral and Maxillofacial Surgery, and ${ }^{5}$ Surgery, Kushiro Rosai Hospital, \\ Hokkaido, Japan; ${ }^{3}$ Department of Neurosurgery, Shin-midori Hospital, Kanagawa, Japan; and ${ }^{6}$ Department \\ of Neurosurgery, Nippon Medical School, Tokyo, Japan
}

\begin{abstract}
Superior cluneal nerve (SCN) entrapment neuropathy is a known cause of low back pain. Although surgical release at the entrapment point of the osteofibrous orifice is effective, intraoperative identification of the thin SCN in thick fat tissue and confirmation of sufficient decompression are difficult. Intraoperative indocyanine green video angiography (ICG-VA) is simple, clearly demonstrates the vascular flow dynamics, and provides real-time information on vascular patency and flow. The peripheral nerve is supplied from epineurial vessels around the nerve (vasa nervorum), and the authors now present the first ICG-VA documentation of the technique and usefulness of peripheral nerve neurolysis surgery to treat SCN entrapment neuropathy in 16 locally anesthetized patients. Clinical outcomes were assessed with the Roland-Morris Disability Questionnaire before surgery and at the latest follow-up after surgery.

Indocyanine green video angiography was useful for identifying the SCN in fat tissue. It showed that the SCN penetrated and was entrapped by the thoracolumbar fascia through the orifice just before crossing over the iliac crest in all patients. The SCN was decompressed by dissection of the fascia from the orifice. Indocyanine green video angiography visualized the SCN and its termination at the entrapment point. After sufficient decompression, the SCN was clearly visualized on ICG-VA images. Low back pain improved significantly, from a preoperative Roland-Morris Questionnaire score of 13.8 to a postoperative score of 1.3 at the last follow-up visit $(\mathrm{p}<0.05)$. The authors suggest that ICG-VA is useful for the inspection of peripheral nerves such as the SCN and helps to identify the SCN and to confirm sufficient decompression at surgery for SCN entrapment.

(http://thejns.org/doi/abs/10.3171/2013.7.SPINE1374)
\end{abstract}

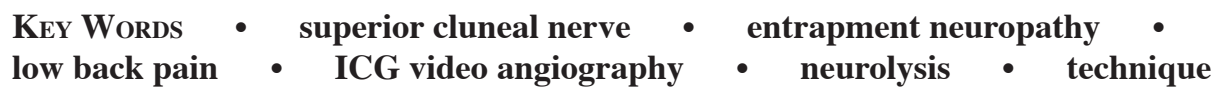

$\mathrm{S}$ INCE Strong and Davila ${ }^{12}$ first reported cluneal nerve syndrome in 1957, SCN entrapment neuropathy has been documented as a cause of LBP. It tends to be misdiagnosed as a lumbar spine disorder and remains poorly understood clinically. There are few reports on surgical techniques to address this entity. ${ }^{4-6,11,12} \mathrm{We}$ developed a less invasive surgical procedure using a microscope to treat SCN entrapment neuropathy under local anesthesia. ${ }^{8}$ However, the intraoperative identification of the thin SCN in the fat layer and confirmation of sufficient decompression can be difficult.

Abbreviations used in this paper: ICG-VA $=$ indocyanine green video angiography; $\mathrm{LBP}=$ low-back pain; $\mathrm{RMDQ}=$ Roland-Morris Disability Questionnaire; SCN = superior cluneal nerve.
The peripheral nerve is supplied by surrounding epineurial vessels (vasa nervorum); insufficient supply from the vasa nervorum results in nerve damage and neuropathy. ${ }^{2,3,14}$ Therefore, for successful neurolysis, nerve decompression and improvement of the blood flow in the vasa nervorum ${ }^{2}$ are both necessary.

Intraoperative ICG-VA clearly demonstrates the vascular flow dynamics and facilitates the selection of appropriate surgical procedures. It is simple and provides real-time information on the patency of arterial and venous vessels and their flow. ${ }^{1,9,10}$ In our search of the literature, we found no documentation of the use of ICG-VA in peripheral nerve neurolysis surgery. Here we present our technique and report on the usefulness of ICG-VA for the neurolysis of SCN entrapment neuropathy. 


\section{Methods}

\section{Our Criteria for the Diagnosis of SCN Entrapment Neuropathy}

Elsewhere ${ }^{8}$ we have proposed criteria for the diagnosis of SCN entrapment neuropathy; the criteria include unilateral LBP involving the iliac crest and buttock, a trigger point over the posterior iliac crest located $7 \mathrm{~cm}$ from the midline (corresponding to the nerve compression zone), and numbness and radiating pain in the SCN area upon compression of the trigger point. While symptom relief in more than $75 \%$ of patients was obtained within 2 hours of inducing local nerve block by injecting $2 \mathrm{ml}$ of $1 \%$ lidocaine at the trigger point in the buttocks, ${ }^{5,6,13}$ some patients reported pain recurrence after the analgesic effect of the test injection wore off.

All patients gave prior written consent for participation in this study. All had undergone unsuccessful conservative treatment including perioral medication and SCN block. We excluded patients in whom pain relief was achieved by conservative treatment. None of the treated patients had undergone iliac crest harvest for grafting, had suffered trauma to the affected area, or reported rapid weight gain associated with the onset of SCN entrapment neuropathy.

\section{Patient Population}

We followed 16 patients with SCN entrapment neuropathy. The 6 men and 10 women, ranging in age from 38 to 82 years (mean 63 years), underwent surgery performed by the senior author (T.I.) between February 2012 and July 2012. The affected side was unilateral in 7 patients and bilateral in 9 patients. The duration of symptoms from onset to treatment averaged 41 months (range 6-168 months). We carefully assessed our patients to ascertain that their LBP was attributable to SCN entrapment. The mean postoperative follow-up period was 12 months (range 9-14 months).

\section{Indocyanine Green Video Angiography}

We performed decompression surgery under a surgical microscope (OPMI Pentero, Carl Zeiss). For intraoperative ICG-VA, we intravenously injected $5 \mathrm{mg}$ ICG as a bolus. ${ }^{9}$ The angiographic images were observed on a video screen in real time and recorded by a video camera attached to the microscope.

\section{Surgical Technique}

As we have reported elsewhere, ${ }^{8}$ with the patient placed prone and under local anesthesia, we performed microsurgical release of the SCN entrapment. We made a 5-cm-long skin incision across the trigger point located $7-8 \mathrm{~cm}$ from the midline on the iliac crest. We then carefully dissected the subcutaneous soft tissue and identified the SCN with a nerve stimulator placed on the fat layer over the subcutaneous space. Because the SCN is thin and buried in a fat layer, its identification can be difficult. In such cases we use ICG-VA because it helps to identify the SCN as a line buried in the fat layer.

The SCN slants from caudolateral to rostromedial and penetrates the thoracolumbar fascia through the orifice just before crossing over the iliac crest. The SCN, observed on ICG-VA images before decompression, appears as a line that stops at the entrapment point. After sharply cutting the thoracolumbar fascia of the orifice with microscissors in a distal to rostral direction along the SCN to release its entrapment, we confirm decompression of the SCN by its posterior bulging. We then cut the thoracolumbar fascia until we reach a point where the $\mathrm{SCN}$ is free of kinks. We reconfirm decompression by looking for a blood vessel on ICG-VA images.

\section{Evaluation of Treatment Outcomes}

Clinical outcomes were assessed with the RMDQ before surgery and at the latest follow-up after surgery. For statistical analysis, we subjected our data to the paired t-test using Statmate III software (ATMS Co. Ltd.). Differences of $\mathrm{p}<0.05$ were considered to indicate statistical significance.

\section{Results}

During surgery for SCN entrapment, we acquired ICG-VA images 3-5 times. When the SCN was identified in a subcutaneous area after dissection, we did not perform ICG-VA. Of $25 \mathrm{SCNs}$, IGC-VA identified 3 that were not found under the microscope. All 25 nerves penetrated the thoracolumbar fascia through the orifice just before crossing over the iliac crest. Superior cluneal nerve entrapment by the fascia was severe in all patients, and the SCN was decompressed by dissection of the fascia from the orifice. We acquired ICG-VA images before and after decompression of the SCN. Indocyanine green video angiography demonstrated not only the vasa nervorum but also normal veins in the fat tissue. Normal veins were observed in the dark field of ICG-VA images. Although the vasa nervorum tended to be recognizable as linear structures, their precise identification required observation under the microscope.

Visualization of the vasa nervorum was improved in all cases upon decompression of the SCN. On ICG-VA images, the appearance of the SCN changed from a faint line before to a clear bright line after complete release of the entrapped SCN. Indocyanine green video angiography showed that of the 16 patients with $25 \mathrm{SCNs}, 4$ required further decompression; 3 of these patients presented with 2 SCN branches and the other with 3 branches.

There were no local or systemic complications during or after the operation. All patients reported symptom improvement after the decompression during surgery; none experienced postoperative worsening of their symptoms. Based on the RMDQ scores, all patients had significant improvement at the last follow-up ( $\mathrm{p}<0.05)$. The RMDQ scores before surgery and at the last follow-up visit were 13.8 and 1.3 , respectively.

\section{Illustrative Cases}

\section{Case 1}

In this 82-year-old man with a 2-year history of LBP, 
several observation therapies had failed and his LBP interfered with his daily activities. He presented at our institute with LBP and had difficulty standing, sitting, and walking. A diagnosis of SCN entrapment neuropathy was made. As SCN block resulted in only transient pain relief and as subsequent treatments failed, we decided to perform surgery.

Despite a careful search, we were unable to find the $\mathrm{SCN}$ in the fat tissue. When we performed ICG-VA, we were then able to identify the SCN as a thin line in the fat tissue (Fig. 1A and B). It penetrated the thoracolumbar fascia through the orifice and was discernible on ICG-VA images (Fig. 1C and D). After opening the orifice with microscissors in the distal to rostral direction along the $\mathrm{SCN}$, the released SCN was clearly visualized on ICGVA images (Fig. 1E and F). Intraoperatively, his radiating pain could not be elicited by manual direct compression of the SCN. His LBP immediately improved after the operation and he suffered no recurrence in the 12 months following this treatment. His RMDQ score improved from 9 of 24 to 2 of 24 at the final follow-up.

\section{Case 2}

This 65-year-old man with a 1-year history of LBP
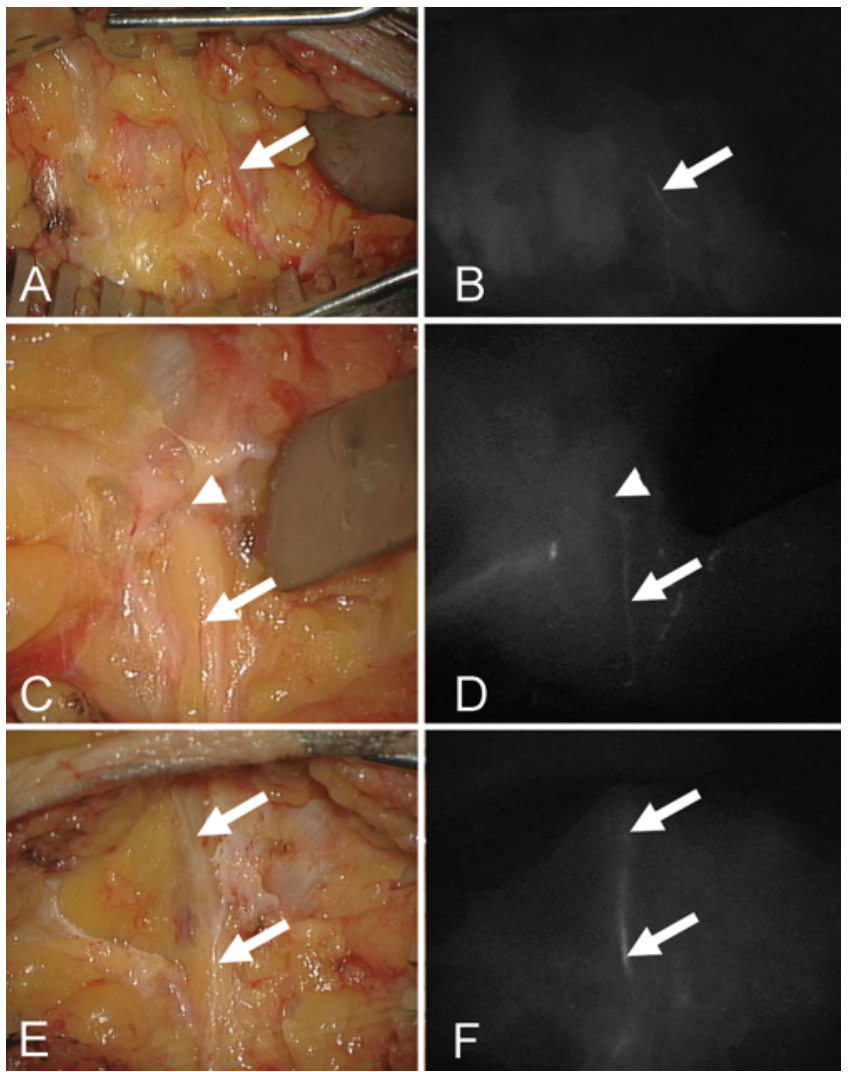

FIG. 1. Case 1. Intraoperative views under a microscope (operative views $[A, C$, and $E]$ and ICG-VA images $[B, D$, and $F])$. $A$ and $B$ : ICG-VA after partial dissection of the fat layer shows the SCN in the fat tissue (arrow). C and D: The SCN penetrated the thoracolumbar fascia through the orifice (arrowhead). ICG-VA shows the SCN (arrow) terminating at the orifice (arrowhead). E and F: The SCN is released by opening the orifice with microscissors in the distal to rostral direction along the SCN. ICG-VA clearly shows the released SCN (arrows). had been conservatively treated elsewhere with medications. However, his pain gradually increased, and he presented at our institute with LBP and had difficulty standing, sitting down, and sitting for prolonged periods. His LBP was diagnosed as SCN entrapment neuropathy. Because SCN block yielded only transient pain relief and subsequent treatments failed, he underwent surgery. We first addressed his right SCN entrapment neuropathy because it produced the greater degree of pain.

The SCN and its branch penetrated the thoracolumbar fascia through the orifice just before crossing over the iliac crest. We opened the orifice with microscissors in the distal to rostral direction along the SCN and performed ICG-VA. The SCN was clearly visualized, but the branch was poorly visualized (Fig. 2A-C). After dissection in the distal direction along the SCN branch, the ICG-VA image showed the released SCN branch clearly (Fig. 2D-F). Intraoperative manual direct compression of the SCN failed to elicit radiating pain. One week later, we released the SCN entrapment on the right side. His LBP improved immediately and he suffered no recurrence in the 11 months following this treatment. His RMDQ score improved from 19 of 24 to 0 of 24 at the final follow-up.

\section{Discussion}

The SCN can be entrapped at the osteofibrous orifice where it penetrates the thoracolumbar fascia and produces LBP. ${ }^{4-8}$ In fact, $1.6 \%{ }^{6}$ to $12 \%{ }^{4}$ of all LBP is due to $\mathrm{SCN}$ entrapment neuropathy. Surgical release at the point where the SCN exits through the osteofibrous orifice has been reported as effective. ${ }^{6,8,11}$ In some cases, intraoperative identification of the SCN is hampered by its thinness and its subcutaneous location after it penetrates through the orifice in an area with much fat tissue. We found that an incision placed at the trigger point and nerve stimulation under a microscope, while time-consuming, aids in the identification of the SCN.

As ICG-VA clearly demonstrates, vascular flow dynamics can help in the selection of appropriate surgical procedures. It is simple, provides real-time information on the patency of arterial and venous vessels and their flow, ${ }^{1,9,10}$ and is a useful adjunct to improve the quality of neurovascular procedures and to document the vascular flow intraoperatively. ${ }^{10}$ Vessels around the peripheral nerve (vasa nervorum) provide its blood supply. In this study, we are the first to document that the vasa nervorum of the peripheral nerve can be visualized on intraoperative ICG-VA images and that they show improved vessel flow around the SCN upon its release from entrapment. Consequently, ICG-VA may be a useful tool in peripheral nerve surgery. It may also be highly useful for the surgical treatment of patients with tibial nerve entrapment at the tarsal tunnel and ulnar nerve entrapment at Guyon's canal. These surgeries are not common and it can be difficult to ascertain complete decompression of thin nerves. In addition, ICG-VA may be of use in more common peripheral nerve surgeries involving comparatively thick nerves such as the median nerve, ulnar nerve, and peroneal nerve.

For the intraoperative determination of sufficient 


\section{Intraoperative use of ICG-VA for SCN entrapment}
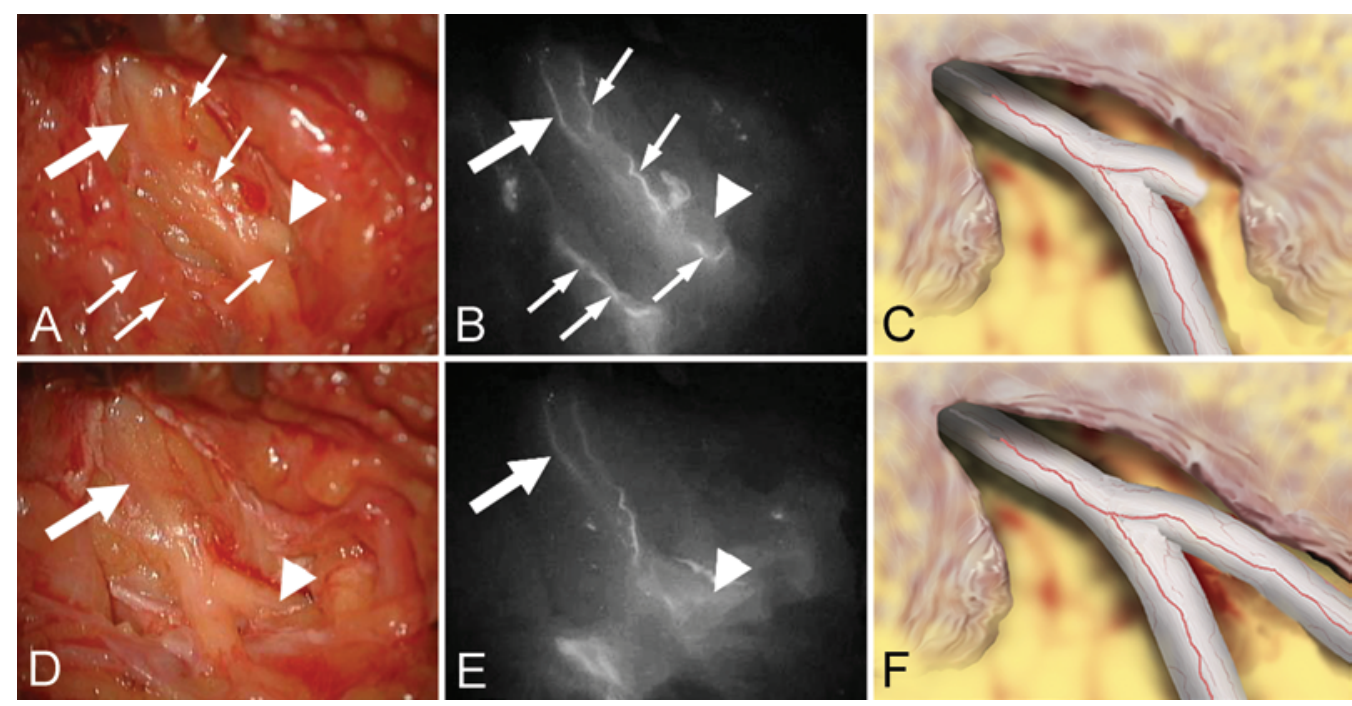

FIG. 2. Case 2. Intraoperative views under a microscope (operative view $[A$ and $D], I C G-V A$ images $[B$ and $E]$, schema of the operative view [C and F]). A-C: The SCN penetrated the thoracolumbar fascia through the orifice. ICG-VA shows the SCN (arrow). The SCN branch is poorly visualized (arrowhead). ICG-VA demonstrates the SCN and the vasa nervorum (arrow) and also normal veins in the fat tissue (small arrows). Although the vasa nervorum tends to be recognized as a linear structure, precise identification requires inspection under the microscope. D-F: The SCN branch is released by dissection in the distal direction (arrow). ICG-VA clearly shows the released SCN branch (arrowhead).

SCN release, we recommended visual confirmation of adequate SCN decompression and the questioning of locally anesthetized patients regarding their sensations. ${ }^{8}$ Nonetheless, other indices of sufficient SCN release are desirable because not all patients recognize pain disappearance immediately and the surgeon's experience is needed for the complete release of the SCN. Moreover, surgical results tend to be unsatisfactory in cases without visible nerve compression, and the entrapped nerve may not be addressed due to the presence of branches. ${ }^{6}$ Based on our experience with ICG-VA, the disturbed blood flow in the vasa nervorum due to entrapment of the $\mathrm{SCN}$ and/ or its branches improves upon vessel release. Ischemia of the peripheral nerve results in nerve damage and peripheral nerves with long axons are fed by the accompanying blood vessels (vasa nervorum) ${ }^{2,3,14}$ Therefore, for the treatment of peripheral nerve anomalies, knowledge about the vasa nervorum supplying nutrients to these nerves is important $;^{2}$ for the release of peripheral entrapment, the nerve structure and criteria for improved nerve blood flow from the vasa nervorum must be understood. Consequently, it is reasonable to confirm improvement in the blood flow of the vasa nervorum upon release from entrapment. Indocyanine green video angiography can demonstrate the vasa nervorum clearly and is the optimal technique to confirm release of peripheral nerve entrapment.

Our study has some limitations. The number of patients was small, and the postoperative follow-up period was relatively short (mean 12 months). Because the diagnostic area of ICG-VA is limited to the field of the surgical microscope and because several structures may interfere with visualization of the $\mathrm{SCN}$, a certain amount of dissection is required. Also, in patients suffering recurrence, the presence of scar tissue may render the subsequent identification of the SCN on ICG-VA images difficult. In addi- tion, as the ICG dye contains iodine, it cannot be used in patients allergic to iodine.

To validate the usefulness and safety of ICG-VA in patients undergoing SCN decompression surgery, cohort studies must be performed. Nonetheless, because ICG-VA is easy and less invasive and provides important information for surgery, we suggest that it is useful in procedures that address peripheral nerve entrapment.

\section{Conclusions}

Our findings suggest that ICG-VA is useful for observing peripheral nerves such as the SCN. For SCN entrapment surgery, ICG-VA helps to identify the SCN and its branches and to confirm sufficient nerve decompression.

\section{Disclosures}

The authors report no conflict of interest concerning the materials or methods used in this study or the findings specified in this paper.

Author contributions to the study and manuscript preparation include the following. Conception and design: Kim, Isu, Ohtsubo, Kusano. Acquisition of data: Kim, Isu, Chiba, Morimoto. Analysis and interpretation of data: Kim, Isu, Ohtsubo, Kusano. Drafting the article: Kim. Critically revising the article: Isu, Ohtsubo, Kusano, Kobayashi, Morita. Reviewed submitted version of manuscript: all authors. Approved the final version of the manuscript on behalf of all authors: Kim. Statistical analysis: Kim. Administrative/technical/ material support: Isu, Ohtsubo, Kusano. Study supervision: Isu.

\section{References}

1. Hanel RA, Nakaji P, Spetzler RF: Use of microscope-integrated near-infrared indocyanine green videoangiography in the surgical treatment of spinal dural arteriovenous fistulae. Neurosurgery 66:978-985, 2010

2. Ishibe K, Tamatsu Y, Miura M, Shimada K: Morphological 


\section{K. Kim et al.}

study of the vasa nervorum in the peripheral branch of human facial nerve. Okajimas Folia Anat Jpn 88:111-119, 2011

3. Kennedy JM, Zochodne DW: Influence of experimental diabetes on the microcirculation of injured peripheral nerve: functional and morphological aspects. Diabetes 51:2233-2240, 2002

4. Kuniya H, Aota Y, Nakamura N, Kawai T, Tanabe H, Saito T: [Low back pain patients with suspected entrapment of the superior cluneal nerve.] J Spine Res 2:1032-1035, 2011 (Jpn)

5. Lu J, Ebraheim NA, Huntoon M, Heck BE, Yeasting RA: Anatomic considerations of superior cluneal nerve at posterior iliac crest region. Clin Orthop Relat Res 347:224-228, 1998

6. Maigne JY, Doursounian L: Entrapment neuropathy of the medial superior cluneal nerve. Nineteen cases surgically treated, with a minimum of 2 years' follow-up. Spine (Phila Pa 1976) 22:1156-1159, 1997

7. Maigne JY, Lazareth JP, Guérin Surville H, Maigne R: The lateral cutaneous branches of the dorsal rami of the thoracolumbar junction. An anatomical study on 37 dissections. Surg Radiol Anat 11:289-293, 1989

8. Morimoto D, Isu T, Kim K, Imai T, Yamazaki K, Matsumoto $\mathrm{R}$, et al: Surgical treatment of superior cluneal nerve entrapment neuropathy. Technical note. J Neurosurg Spine 19:7175,2013

9. Murakami T, Koyanagi I, Kaneko T, Iihoshi S, Houkin K: Intraoperative indocyanine green videoangiography for spinal vascular lesions: case report. Neurosurgery 68 (1 Suppl Operative):241-245, 2011

10. Raabe A, Beck J, Gerlach R, Zimmermann M, Seifert V: Near- infrared indocyanine green video angiography: a new method for intraoperative assessment of vascular flow. Neurosurgery 52:132-139, 2003

11. Speed S, Sims K, Weinrauch P: Entrapment of the medial branch of the superior cluneal nerve. A previously unrecognized cause of lower back pain in cricket fast bowlers. J Med Cases 2:101-103, 2011

12. Strong EK, Davila JC: The cluneal nerve syndrome; a distinct type of low back pain. Ind Med Surg 26:417-429, 1957

13. Tubbs RS, Levin MR, Loukas M, Potts EA, Cohen-Gadol AA: Anatomy and landmarks for the superior and middle cluneal nerves: application to posterior iliac crest harvest and entrapment syndromes. Laboratory investigation. J Neurosurg Spine 13:356-359, 2010

14. Utuk A, Sarikcioglu L, Demirel BM, Demir N: The immunosuppressive agent FK506 prevents subperineurial degeneration and demyelination on ultrastructural and functional analysis. Curr Neurovasc Res 6:252-258, 2009

Manuscript submitted January 23, 2013.

Accepted July 30, 2013.

Please include this information when citing this paper: published online September 20, 2013; DOI: 10.3171/2013.7.SPINE1374.

Address correspondence to: Kyongsong Kim, M.D., Ph.D., Department of Neurosurgery, Chiba Hokuso Hospital, Nippon Medical School, 1715 Kamagari, Inzai City, Chiba 270-1694, Japan. email: kyongson@nms.ac.jp. 